\title{
Demo: CMOS-SPAD Camera Prototype for Single-Sensor 2D/3D Imaging
}

\author{
I. Vornicu \\ Instituto Microelectrónica Sevilla \\ IMSE-CNM \\ CSIC-Universidad de Sevilla (Spain) \\ ivornicu@imse-cnm.csic.es
}

\author{
R. Carmona-Galán \\ Instituto Microelectrónica Sevilla \\ IMSE-CNM \\ CSIC-Universidad de Sevilla (Spain) \\ rcarmona@imse-cnm.csic.es
}

\author{
Á. Rodríguez-Vázquez \\ Instituto Microelectrónica Sevilla \\ IMSE-CNM \\ CSIC-Universidad de Sevilla (Spain) \\ angel@imse-cnm.csic.es
}

\begin{abstract}
One of the research lines explored in project 'iCaveats' has been the combined capture of 2D and 3D visual information. With the objective of power-efficient feature learning/extraction, combined $2 \mathrm{D} / 3 \mathrm{D}$ imaging is a useful tool to work on a lightweight but rich description of the scene. Single-sensor capture of both modalities is a potential improvement in cost and efficiency. In this demo, we present the performance and features of a CMOS-SPAD camera prototype that realizes photon counting and direct time-offlight (d-ToF). The central elements of the camera module are a $64 \times 64$ SPAD imager and a FPGA board for real time histograming and image reconstruction at $1 \mathrm{kfps}$.
\end{abstract}

\section{CCS Concepts}

- Hardware $\rightarrow$ Communication hardware, interfaces and storage $\rightarrow$ Sensor devices and platforms.

\section{Keywords}

2D/3D image reconstruction, direct time-of-flight, CMOS-SPAD image sensor, time gating, low illumination.

\section{INTRODUCTION}

The possibility of acquiring 2D and 3D images from a single device represents a reduction in size, power consumption and complexity in embedded vision systems. Traditional methods for 3D imaging, i. e. triangulation/stereo vision, require many computational resources. The capture and integration of $2 \mathrm{D}$ and $3 \mathrm{D}$ information in the same silicon substrate facilitates mapping between the two views. A procedure that is much more complex in terms of computations in stereo vision. For this reason, estimation of the time-of-flight (ToF) is very much wanted. Some attempts to indirect ToF estimation rely on photonic mixers to demodulate the illumination signal [1], what requires access to special fabrication steps. Some others rely on charge integration [2], what requires very precise charge transfers for accurate background cancellation. This is usually achieved through intervention in the fabrication process doping profiles.

An interesting alternative in CMOS technology are avalanche diodes in Geiger mode, also known as single-photon avalanche diodes (SPAD). These devices are sensitive to the arrival of a single-photon. These events can be counted, thus providing an

\footnotetext{
Permission to make digital or hard copies of part or all of this work for personal or classroom use is granted without fee provided that copies are not made or distributed for profit or commercial advantage and that copies bear this notice and the full citation on the first page. Copyrights for third-party components of this work must be honored. For all other uses, contact the Owner/Author. Copyright is held by the owner/ author(s).

ICDSC '18, September 3-4, 2018, Eindhoven, Netherlands

(C) 2018 Copyright is held by the owner/author(s).

ACM ISBN 978-1-4503-6511-6/18/09

https://doi.org/10.1145/3243394.3243711
}

intensity map, i. e. 2D image, even in low-illumination conditions; or they can be time stamped with the help of a fine time-to-digital converter (TDC) for direct estimation of the ToF [3].

\section{VISITOR EXPERIENCE}

The principle of d-ToF is explained in the diagram of Fig. 1(a). Short light pulses are sent by a picosecond laser. These pulses are reflected by objects situated at image planes between $P_{i}$ and $P_{f}$. At the same time, an electrical signal is sent to the global shutter of the imager. Some of the outgoing photons reflected by the object reach the active surface of the sensor and are eventually detected.

In this demo, the operation of the 2D/3D camera will be presented with the help of a poster and recorded videos. The d-ToF system is composed by the SPAD camera itself and a picosecond laser (Fig. 1(b)). The housing of the camera accommodates 8mm F1.2 lenses focusing on the $64 \times 64$ array of SPADs and pixel-level TDCs [4]. The control signals are provided by an FPGA. It also implements a real time $2 \mathrm{D}$ and $3 \mathrm{D}$ image reconstruction and a USB link used for image streaming and camera configuration. A user-friendly GUI has been built in Matlab and OpenCV. The camera also has the possibility to record videos at $1000 \mathrm{fps}$ and sent them to the computer in raw format. Afterwards the user can play them off-line at $50 \mathrm{fps}$.

Different experiments are featured in the videos:

$2 D$ imaging: The scene is illuminated by a $30 \mathrm{~W}$ lamp. This amount of light is enough to ensure the minimum count rate required at the input of the CMOS counters which are driven by the SPAD detectors. The brightness map is obtained by counting photons. This is done at pixel-level by a gated $8 \mathrm{~b}$ counter which is actually the coarse counter used by the TDC for 3D imaging. The $2 \mathrm{D}$ image obtained by averaging 100 inter-frames. The frame rate of the reconstructed images is about 10fps.

$3 D$ ranging: We have employed a panel to provide a homogenous background for the shapes to be reconstructed. This panel has been shifted towards the sensor-laser ensemble from $74 \mathrm{~cm}$ down to $4 \mathrm{~cm}$ with $1 \mathrm{~cm}$ step (see Fig. 1). ToF estimation is done in parallel by means of in-pixel TDCs. These TDCs have a $145 \mathrm{ps}$ LSB. In addition, time-gating of the SPADs mitigates the influence of spurious avalanches. The depth map is obtained from the aggregated readings of the pixel ToF estimations. 65k interframes are employed to generate this map off-chip at $1000 \mathrm{fps}$. The SNR of the ToF measurement is about $24 \mathrm{~dB}$. The total jitter FWHM is about 735 ps.

High-speed burst capture: The high-speed photon counting capabilities of the sensor and the camera system memory allows to capture a burst at $1000 \mathrm{fps}$, that can be then downloaded off-line and played in slow motion. 


\section{CONCLUSIONS}

A camera prototype for 2D/3D image reconstruction based on photon counting and $\mathrm{d}-\mathrm{ToF}$ has been developed in this project. It demonstrates that single-sensor $2 \mathrm{D} / 3 \mathrm{D}$ imaging is possible in CMOS technology. However noise and background illumination, translated into spurious avalanches, can seriously degrade image quality. The overall frame rate is limited by the large number of inter-frames needed and image serialization. On-chip image reconstruction in real-time and parallel readout channels can help overcome these limitations.

\section{ACKNOWLEDGMENTS}

This work was mainly supported by the Spanish MINECO and the European Region Development Fund (ERDF/FEDER) through project 'iCaveats' (Ref. TEC2015-66878-C3-1-R), and partially supported by Junta de Andalucía through project 'SmartCIS3D' (Ref. TIC 2338-2013) and by EU-REA through project 'Achieve' (EU H2020 MSCA-ITN 2017, Grant No. 765866).

\section{REFERENCES}

[1] C.S. Bamji et al., "A 0.13 um CMOS System-on-Chip for a 512 x 424 Time-of-Flight Image Sensor With MultiFrequency Photo-Demodulation up to $130 \mathrm{MHz}$ and $2 \mathrm{GS} / \mathrm{s}$ ADC", IEEE JSSC, vol. 50, no. 1, pp. 303-319 (2015).

[2] D. Durini, W. Brockherde, W. Ulfig and B. J. Hosticka, "Time-of-Flight 3-D Imaging Pixel Structures in Standard CMOS Processes." IEEE Journal of Solid-State Circuits, vol. 43, no. 7, pp. 1594-1602 (2008).

[3] F. Villa, R. Lussana, D. Bronzi, et al., "CMOS imager with 1024 SPADs and TDCs for single-photon timing and 3-D time-of-flight", J. of Selected Topics in Quantum Electronics, Vol. 20, No. 6 (2014).

[4] I. Vornicu, R. Carmona-Galán, and Á. Rodríguez-Vázquez, "Arrayable Voltage-Controlled Ring-Oscillator for Direct Time-of-Flight Image Sensors". IEEE TCAS-I, Vol. 64, No. 11, pp. 2821-2834 (2017).

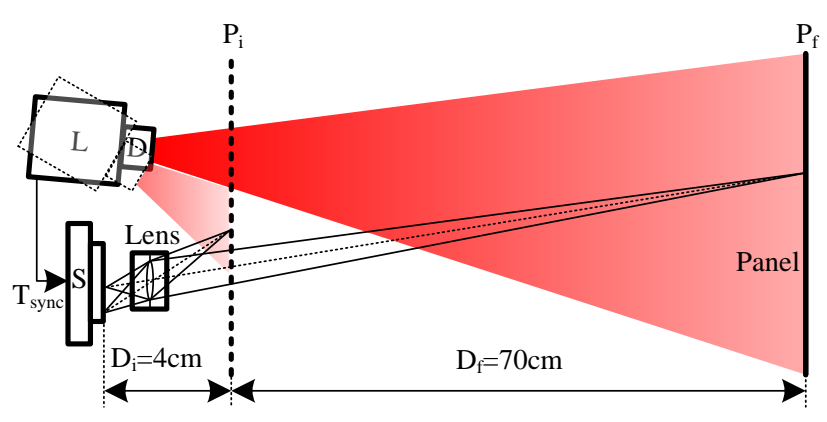

(a)

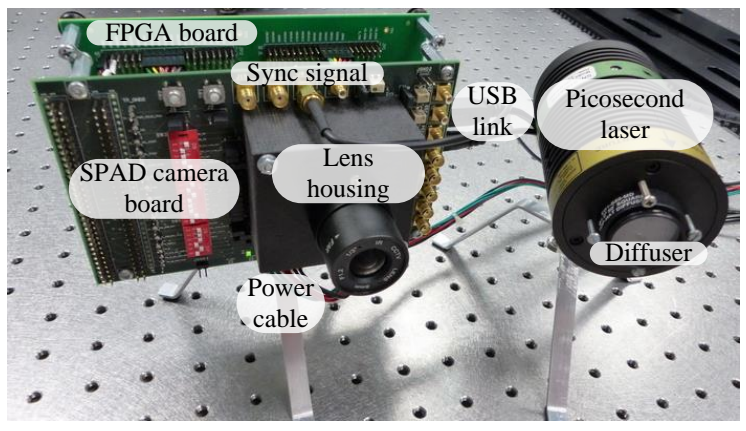

(b)

Fig. 1. (a) Top view of the setup ( $\mathrm{L}=$ picosecond laser; $\mathrm{D}=$ diffuser; $\mathrm{S}=\mathrm{SPAD}$ image sensor) and (b) prototype d-ToF system.

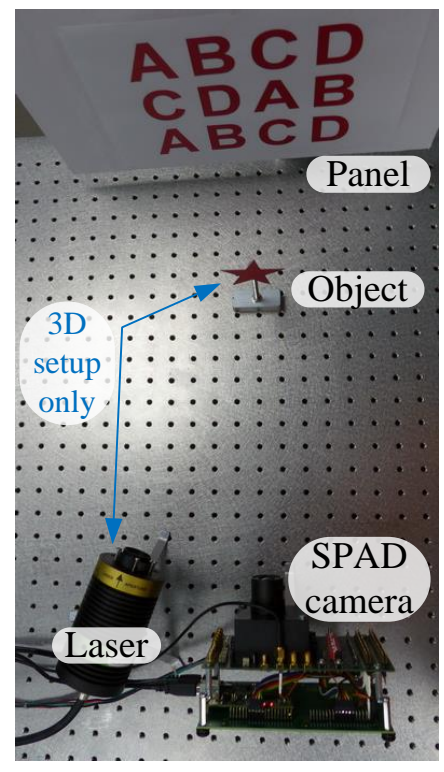

(a) 2D/3D setup
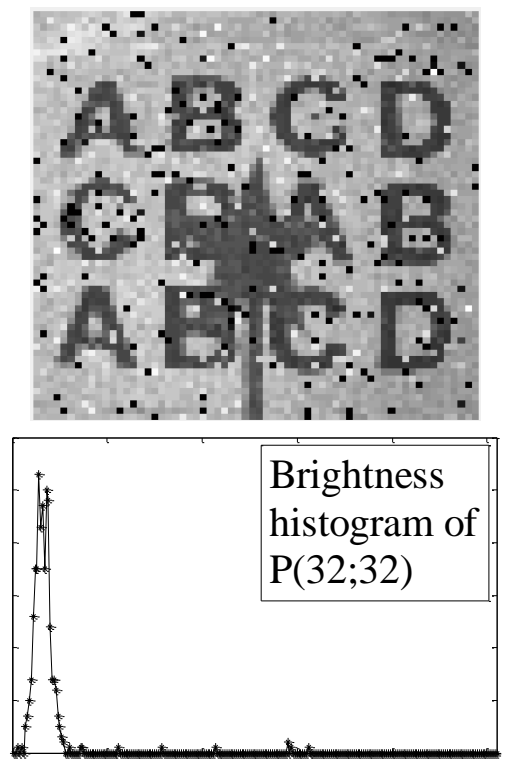

(b) 2D snapshot
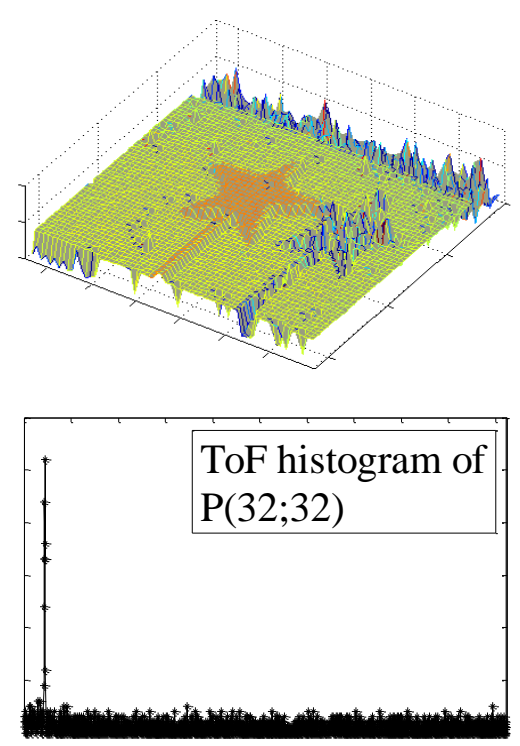

(c) 3D snapshot

Fig. 2. Experimental setup and 2D/3D snapshots of the same scene. 